\title{
INSUFICIÊNCIA RENAL CRÔNICA EM LABRADOR ASSOCIADA AO USO DE ANTI-INFLAMATÓRIOS - RELATO DE CASO
}

\section{CHRONIC RENAL INSUFFICIENCY IN LABRADOR ASSOCIATED WITH THE USE OF ANTI-INFLAMMATORIES - CASE REPORT}

Nadja Soares Vila Nova*I, Renata Celis dos Santos Chagas II, Francisca Manuela de Sousa Freire ${ }^{\text {III }}$, Sthefany Kristinne Alves de Melo ${ }^{I I I}$, Marcos Wanderson Vieira Monteiro III

Resumo. O diagnóstico da Insuficiência Renal Crônica (IRC) inclui a identificação de importantes quadros como azotemia, ou outras alterações que afetem o funcionamento da filtração renal, alterando, assim, a homeostase. Um cão Labrador, macho, 13 anos, $28 \mathrm{~kg}$ foi atendido em uma Clínica Veterinária de Natal, Rio Grande do Norte. Na anamnese foi relatado que o animal tinha dores articulares crônicas, atrofia dos membros torácicos e histórico de descompressão de vértebras lombares e artrodese do joelho direito, além de fazer uso recorrente de anti-inflamatórios (meloxicam) por parte dos tutores. Foram solicitados hemograma, bioquímicos séricos e ultrassonografia de abdômen e estes indicaram quadro grave de azotemia (Ureia: 293, $85 \mathrm{mg} / \mathrm{dL}$ e Creatinina: $5,78 \mathrm{mg} / \mathrm{dL}$ ) e imagem compatível com bexiga neurogênica. Administrou-se fluido Ringer com Lactato, Tramadol, Dipirona, Hidróxido de Alumínio e Ranitidina. Após 7 dias de internação, o animal apresentava dor ao andar e urinar, dificuldade no esvaziamento da bexiga, não se alimentava nem bebia água. Devido à persistência da azotemia (Ureia: 148,39 mg/dL e Creatinina: 5,03 mg/dL) o animal foi eutanasiado. $O$ uso de anti-inflamatórios ao longo dos anos levou à insuficiência renal crônica.

PALAVRAS-CHAVE: Insuficiência Renal. Anti-Inflamatórios. Ureia. Creatinina.

Abstract. The diagnosis of Chronic Kidney Disease (CKD) includes the identification of important conditions such as azotemia or other changes that affect the functioning of kidney filtration, thus altering homeostasis. A Labrador dog, male, 13 years old, 28 kg was treated at a Veterinary Clinic in Natal, Rio Grande do Norte. In the anamnesis, it was reported that the animal had chronic joint pain, atrophy of the thoracic limbs and a history of decompression of the lumbar vertebrae and arthrodesis of the right knee, in addition to the constant use of anti-inflammatory drugs by its tutors. A complete hemogram, a serum biochemistry and an abdominal ultrasound were requested and indicated severe azotemia (Urea: $293.85 \mathrm{mg} / \mathrm{dL}$ and Creatinine: $5.78 \mathrm{mg} / \mathrm{dL}$ ) and a neurogenic bladder compatible image. Lactated Ringer's solution, Tramadol, Dipyrone, Aluminum Hydroxide and Ranitidine were administered. After 7 days of hospitalization, the animal presented pain when walking and urinating, difficulty in emptying the bladder, did not eat nor drink water. Due to the persistence of azotemia (Urea: $148.39 \mathrm{mg} / \mathrm{dL}$ and Creatinine: $5.03 \mathrm{mg} / \mathrm{dL}$ ) the animal was euthanized. The use of anti-inflammatory medication over the years led to chronic kidney disease.

KEYWORDS: Renal Insufficiency. Anti-inflammatory Medication. Urea. Creatinine.

'Doutora em Medicina Veterinária. Docente, Faculdade de enfermagem Nova Esperança. Departamento de Medicina Veterinária - FACENE. *Autor correspondente: nadja.vilanova@hotmail.com. CEP: 58067-695, João Pessoa, Paraíba, Brasil. ORCID ID: 0000-0003-1905-9961.

"Médica Veterinário. Clínica Veterinária Cidade Jardim. CEP: 59078-340, Natal, Rio Grande do Norte, Brasil. ORCID ID: 0000-0003-0561-0951.

III Discente de Medicina Veterinária. Faculdade de Enfermagem e Medicina Nova Esperança - FACENE. CEP: 58067-695, João Pessoa, Paraíba, Brasil. ORCID ID: 0000-0003-3486-4272; 0000-0003-3323-4105; 0000-0001-8373-8665. 


\section{INTRODUÇÃO}

Os rins são órgãos especializados em filtrar o sangue através de unidades funcionais denominadas de néfron. Nelas existem os chamados corpúsculos renais, formados pelos glomérulos e cápsulas de Bowman, e túbulos que desembocam em um ducto coletor. ${ }^{1}$

O principal motivo das investigações do sistema urinário, através de análises séricas em exames bioquímicos e/ou urinálises, é reconhecer se existe alguma falha, seja aguda ou crônica. ${ }^{2}$ o processo inclui a identificação de quadros como o de azotemia, causado pelo aumento nas concentrações de ureia e creatinina. Qualquer alteração que afete o funcionamento na atividade dos néfrons, quebrando assim a homeostase, dá origem a um quadro de insuficiência renal, caracterizado por lesão do tipo crônica ou aguda, podendo evoluir até o óbito do animal. ${ }^{1,3,4,5}$

Órgãos internacionais da saúde como a IRIS (International Renal Interest Society, 2019) ${ }^{6}$ estabelecem critérios e tabelam valores para auxiliar os médicos veterinários na construção do diagnóstico de paciente nefropatas. Assim, a insuficiência renal crônica pode ser dividida em até quatro estágios diferentes de acordo com os valores de creatinina sérica apresentados nos exames bioquímicos dos animais e o tratamento deverá ser feito de acordo com o estágio deste paciente. Uma das medidas a ser tomada por estes tratamentos é a retirada

\section{DESCRIÇÃO DO CASO}

Um canino macho, da raça Labrador, treze anos de idade, pesando de algum tipo de medicação que possa vir a ter um efeito colateral nefrotóxico, como os anti-inflamatórios não esteroides (AINES).

A classe de fármacos dos AINES atua no organismo animal como um mecanismo inibitório das cicloxigenases causando, consequentemente, diminuição nos níveis de prostaglandinas, prostaciclinas e tromboxanos, substâncias que são mediadoras do processo inflamatório. ${ }^{7}$ São fármacos bastantes utilizados na rotina clínica de atendimento aos pequenos animais, por inibirem os dois grupos das cicloxigenases: COX-1 e COX-2. Os AINES podem atuar tanto no sistema nervoso central quanto no periférico, proporcionando efeitos não só anti-inflamatórios, mas também analgésicos, antitrombóticos e antipiréticos. ${ }^{8,9}$

Entretanto, a homeostase renal depende da atuação dessas cicloxigenases para suas atividades como autorregulação de água e de suas funções tubulares, logo a inibição da COX-2, por exemplo, irá afetar os índices de perfusão renal, causar e/ou agravar lesão renal, retenção sódica e diminuição nos níveis de função tubular. ${ }^{10}$

Visando contribuir com o conhecimento acadêmico da área, o presente trabalho tem como objetivo relatar um caso de insuficiência renal crônica em um canino, labrador, desenvolvida através do uso prolongado de anti-inflamatórios não esteroides, devido ao quadro de problemas articulares. 
Ele apresentava um quadro de otite, queixa principal por parte da tutora, e falta de apetite. Durante a anamnese, foi relatado que o animal possuía múltiplos problemas articulares, tendo feito uma descompressão de vértebras lombares quando possuía oito anos de idade e um procedimento de artrodese, no membro pélvico direito, feito um ano após a descompressão.

No exame físico, foi observado que o paciente apresentava dores articulares nos membros pélvicos e atrofia dos membros torácicos devido à falta de movimentação. $\mathrm{O}$ paciente possuía um histórico de consumo do medicamento anti-inflamatório meloxicam

\section{DISCUSSÃO}

O hemograma (Tabela 1) revelou um quadro de anemia normocítica normocrômica e alterações nas taxas de proteína total e proteinúria. Ainda é muito incerto relacionar essa taxa elevada com surgimento de alguma doença renal em pacientes clinicamente saudáveis, porém já se sabe que níveis
(AINE) por um período de 10 anos. Por ser um fármaco de fácil acesso, ou seja, de não exigir prescrição para sua compra, os tutores do animal administravam o medicamento sempre que ele apresentava sinais de dores nas articulações, não havendo, portanto, um acompanhamento periódico por parte do profissional médico veterinário.

Foram solicitados exames complementares para o quadro do paciente: hemograma, bioquímicos séricos (TGP, TGO, Creatinina, Fosfatase Alcalina, Ureia, Proteína Total, Fósforo, Cálcio, Sódio e relação $\mathrm{PU} / \mathrm{CU}$ ) e uma ultrassonografia de abdome.

elevados de proteína em um paciente com suas funções renais comprometidas podem vir a agravar o caso. ${ }^{1} \mathrm{~A}$ proteinúria é um importante achado clínico na esfera das doenças renais, tendo como fundamental ferramenta o exame de urinálise. ${ }^{2}$

TABELA 1: Resultados do hemograma do paciente, cão macho da raça Labrador, treze anos de idade. Rio Grande do Norte, 2019

\begin{tabular}{cccc}
\hline Hemograma & $\mathbf{0 5 / 0 1 / 2 0 1 9}$ & $\mathbf{1 0 / 0 1 / 2 0 1 9}$ & Valores de Referência \\
\hline \hline Série Vermelha & & & \\
\hline Hemácias & 4,0 & 5,6 & $6,4-7,7$ \\
Hemoglobina & 8,0 & 11,3 & $15,8-19,0$ \\
Hematrócito & 24 & 34 & $30-39$ \\
VCM & 60,0 & 60,8 & $60,0-72,0$ \\
HCM & 20,0 & 20,2 & $20-24$ \\
CHCM & 33,4 & 33,3 & $30-37$ \\
Plaquetas & 208 & 232 & $200-500$ \\
Proteína Total & 8,6 & 8,0 & $6,0-8,0$ \\
\hline Séria Branca & & & \\
\hline Leucócitos Totais & 8.000 & 5.400 & $6.000-13.000$ \\
Mielócitos & 00 & 00 & 00 \\
Metamielócitos & 00 & 00 & 00 \\
Bastão & 00 & 00 & $0-150$ \\
Segmentado & 6.320 & 4.860 & $3.500-10.000$ \\
Eosinófilos & 400 & 108 & $200-1000$ \\
Basófilos & 00 & 00 & $0-150$ \\
Linfócitos & 1.280 & 432 & $1.000-4.500$ \\
Monócitos & 00 & 00 & $200-1.000$ \\
\hline
\end{tabular}


Nos exames bioquímicos (Tabela 2) foi detectada grande alteração nos níveis de ureia e creatinina. Uma das funções primordiais do sistema urinário é a excreta dessas duas substâncias, sendo a creatinina um dos principais biomarcadores. ${ }^{2,11}$ Quando essa função é comprometida os níveis séricos dessas substâncias aumentam, caracterizando um quadro de azotemia, comumente visto em pacientes com insuficiência renal.

TABELA 2: Resultados dos exames bioquímicos do paciente, cão macho da raça Labrador, treze anos de idade. Rio Grande do Norte, 2019

\begin{tabular}{ccccc}
\hline Bioquímicos & $\mathbf{0 5 / 0 1 / 2 0 1 9}$ & $\mathbf{1 0 / 0 1 / 2 0 1 9}$ & $\mathbf{1 1 / 0 1 / 2 0 1 9}$ & Valores de Referência \\
\hline \hline TGP (ALT) & 38,93 & - & - & $21-102$ \\
TCO (AST) & 26,01 & - & - & $23-66$ \\
Creatinina & 5,78 & 6,53 & - & $0,5-1,5$ \\
Fosfatase Alcalina & 45,33 & - & - & $20-156$ \\
Ureia & 293,85 & 198,86 & - & $21,4-59,5$ \\
PU/CU & - & 33,3 & 1,56 & $0,0-0,5$ \\
Cálcio & - & 12,2 & 12,2 & $9,0-11,3$ \\
Fósforo & - & 10,02 & - & $2,6-6,8$ \\
Potássio & - & - & 4,1 & $4,37-5,35$ \\
Sódio & - & - & 152,0 & $140,0-152,0$ \\
\hline
\end{tabular}

Um dos sinais clínicos que acompanha o processo inflamatório é a dor, portanto, o uso de medicações que possam aliviar esse sinal é de fundamental importância no tratamento do paciente e os AINES da subclasse dos "oxicam" atuam no tratamento de dores musculoesqueléticas e articulares. ${ }^{12,13}$

O paciente possuía um histórico de uso do medicamento meloxicam por mais de 10 anos, sendo o mesmo administrado pelos tutores sem o acompanhamento periódico do profissional médico veterinário. A automedicação é caracterizada como o ato da utilização de um determinado produto, visando o alívio de uma sintomatologia. ${ }^{14}$ Entretanto, para que o fármaco tenha seu aproveitamento de forma correta, sua dose, período de tratamento e terapêutica são fatores que precisam ser respeitados. ${ }^{15,16}$

Estudos prévios mostram que uma das classes mais utilizadas de forma indiscriminada por parte de tutores de pequenos animais são os anti-inflamatórios, ${ }^{17,18}$ o que pode ser visto também nesse trabalho.

O anti-inflamatório não esteroide inibidor seletivo para COX-2, meloxicam, pode ter sido um dos fatores para o desenvolvimento e consequente agravamento do quadro, pois uma das causas mais comuns de insuficiência renal é o uso de substâncias nefrotóxicas que agridem o parênquima renal. ${ }^{11} \mathrm{O}$ quadro progressivo de injúria renal do paciente confirma o visto em estudos já publicados $^{10,19,20}$, nos quais o grupo tratado com inibidor de COX-2, durante o período de vinte dias, apresentou injúria renal com queda significativa nos índices de filtração glomerular, efeito adverso que tem sido associado ao uso dos AINES inibidores de COX-2. ${ }^{10}$ Esse efeito inibitório pode vir a causar injúria renal, levando o animal a um consequente estado de proteinúria. $^{20}$

Um quadro de hiperfosfatemia pôde ser evidenciado através dos resultados presentes nos exames bioquímicos (Tabela 2). A alteração dos níveis de fosfato excretado na urina é comum em pacientes nefropatas. ${ }^{1}$ Hiperfosfatemia com taxas excedendo de $10 \mathrm{mg} / \mathrm{dL}$ pode contribuir com o agravo das lesões já existentes no parênquima renal, pois isso irá gerar uma mineralização e intoxicação das unidades nefríticas. ${ }^{1}$

Pacientes hipertensos (pressão arterial sistólica maior que $180 \mathrm{mmHg}$ ) agravam o quadro de insuficiência renal, pois se tornam mais propensos a crises urêmicas 
do que animais saudáveis. ${ }^{1} \mathrm{O}$ paciente não era hipertenso, ou pelo menos não se tinha conhecimento, uma vez que não foram realizados exames específicos como aferição da pressão arterial. Todavia, apresentou níveis limítrofes de sódio no exame bioquímico (152,0 $\mathrm{mEq} / \mathrm{L})$. O excesso de sódio tende a agravar

\section{CONSIDERAÇÕES FINAIS}

Observou-se no presente trabalho que o uso excessivo de anti-inflamatórios por longo período, mesmo que visando o bem-estar do animal, sem acompanhamento clínico, pode levar ao desenvolvimento e/ou agravamento de injúria renal irreversível com progressão do paciente ao óbito. Portanto, é

\section{REFERÊNCIAS BIBLIOGRÁFICAS}

1. Jericó MM. Tratado de medicina interna de cães e gatos. 2 v. 1ed. São Paulo: Roca, 2015.

2. Thrall MA, Weiser G, Allison RW, Campbell TW et al. Hematologia e bioquímica clínica veterinária. São Paulo: Roca Ltda, 2012.

3. Fighera RA, Souza TM, Silva MC, Brum JS, Graça DL, Kommers GD et al. Causas de morte e razões para eutanásia de cães da Mesorregião do Centro Ocidental Rio-Grandense (19652004). Pesq. Vet. Bras. 2008; 28(4): 223-30.

4. Trapp SM, lacuzio AI, Barca Junior FA, Kemper B, Silva LC, Okano W et al. Causas de óbito e razões para eutanásia em uma população hospitalar de cães e gatos. Braz. J. Vet. Res. Anim. Sci. 2010; 47(5): 395-02.

5. Fleming JM, Creevy KE, Promislow DEL. Mortality in North American Dogs from 1984 to 2004: An investigation into age, size and breed- o quadro do paciente com insuficiência renal, pois o animal tem dificuldade, ou não consegue manter o equilíbrio sódico e, em consequência disso, há um aumento tanto na pressão arterial sistólica quanto na pressão intraglomerular, proporcionando agravos nas lesões já presentes no parênquima renal.

de suma importância o acompanhamento de um Médico Veterinário, através de consultas de rotina e a realização de exames clínicolaboratoriais periódicos, que visem avaliar os possíveis sistemas afetados pelas medicações potencialmente tóxicas.

related causes of death. J. Vet. Intern. Med. 2011; 25: $187-98$.

6. IRIS Kidney - Guidelines - IRIS Staging of CKD [Internet]. UK. Disponível em: http://www.iriskidney.com/pdf/IRIS_Pocket_Guide_to_CKD. pdf. 2019

7. Jericó MM, Andrade SF. Antiinflamatórios. In: ANDRADE SF. Manual de Terapêutica Veterinária. 3. ed. São Paulo: Roca, 2008. Cap.7, p.115-40.

8. Kummer CL, Coelho TC. Cyclooxygenase -2 inhibitors nonsteroid anti-inflammatory drugs: current issues. Rev Bras Anestesiol. 2002; 52(4): 98-12.

9. Perkowski SZ, Wetmore LA. The science and art of analgesia. In: Gleed RD \& Ludders JW. Recent Advances in Veterinary Anesthesia and Analgesia: Companion animals. Ithaca: International Veterinary Information Service. 2006. 
10. Papich MG. Un update on nonsteroidal antiinflammatory drugs (NSAIDS) in small animals. VCNA: SAP. Philadelphia. 2008; 38: 1243-66.

11. Kaneko JJ, Harvey JW, Bruss M L. (Ed.). Clinical biochemistry of domestic animals. Academic press, 2008.

12. Luna SP, Basílio AC, Steagall PV, Machado LP, Moutinho FK, Takahira RK et al. Evaluation of adverse effects of longterm oral administration of carprofen, etodolac, flunixin meglumine, ketoprofen, and meloxicam in dogs. AJVR. 2007; 68(3): 258-64.

13. Epstein M, Rodan I, Griffenhagen G, Kadrlik J, Petty M., Robertson S et al. Pain Management Guidelines for Dogs and Cats. JAAHA. 2015; 51(2): 67-84.

14. Quessada AM, Carvalho RL, Klein RP, Silva FAN, Fonseca LS, Miranda DFH, et al.. Uso de medicamentos sem prescrição médico veterinária - comunicação. VN. Uberlândia. 2010; 1(1 e 2): 69-71.

15. Nicoletti MA, Oliveira júnior MA, Bertasso CC, Caporossi PY, Tavares APL.. Principais interações no uso de medicamentos fitoterápicos. Informa. 2007; 19(1 e 2): 32-40.
16. Rocha ALR. Uso racional de medicamentos. 2014. 5op. Monografia (Pós- Graduação em tecnologias Industriais Farmacêuticas) Instituto de tecnologias em Fármacos, Fundação Oswaldo Cruz, Rio de Janeiro, 2014.

17. Zielke M, Carvalho LF, Salame JP, Barbosa DV, Gaspar LFJ, Sampaio LCL. Avaliação do uso de fármacos em animais de companhia sem orientação profissional. SAH. Pelotas - RS. 2018; 6(1): $29-46$.

18. Hansen DTK. Prevalência de intoxicações de cães e gatos em Curitiba. 2006. 72p.. Dissertação (Mestrado em Ciências Veterinárias) - Universidade Federal do Paraná, Curitiba - PR, 2006.

19. Borges $M$, Melchert $A$, Marini Filho $R$, Laposy CB, Alegre BCCP, Silva KR. Função renal, hepática e cardíaca de cães hígidos sob terapia prolongada com celecoxibe. CR. 2012; 42(10) 1844-50.

20. Audia P, Feinfeld DA, Dubrow A, Winchester JF. Metformina induzida acidose láctica e pancreatite aguda precipitada pelo celecoxib, diurético, e candesartan associado a disfunção renal aguda. CT, New York. 2008; 46(2): 164-66. 